\title{
Author Correction: The nature of spin excitations in the one-third magnetization plateau phase of $\mathrm{Ba}_{3} \mathrm{CoSb}_{2} \mathrm{O}_{9}$
}

\author{
Y. Kamiya ${ }^{1}$, L. Ge ${ }^{2}$, Tao Hong (10 ${ }^{3}$, Y. Qiu ${ }^{4}$, D.L. Quintero-Castro ${ }^{5}$, Z. Lu ${ }^{5}$, H.B. Cao ${ }^{3}$, M. Matsuda ${ }^{3}$, E.S. Choi ${ }^{6}$, \\ C.D. Batista (10 ${ }^{7,8}$, M. Mourigal (1) ${ }^{2}$, H.D. Zhou ${ }^{6,7}$ \& J. Ma ${ }^{7,9,10}$
}

Correction to: Nature Communications; https://doi.org/10.1038/s41467-018-04914-1; published online 10 July 2018

The original version of this Article omitted the following from the Acknowledgements:

'J. Ma's primary affiliation is Shanghai Jiao Tong University.'

This has been corrected in both the PDF and HTML versions of the Article.

Published online: 01 August 2018

\begin{abstract}
(c) (i) Open Access This article is licensed under a Creative Commons Attribution 4.0 International License, which permits use, sharing, adaptation, distribution and reproduction in any medium or format, as long as you give appropriate credit to the original author(s) and the source, provide a link to the Creative Commons license, and indicate if changes were made. The images or other third party material in this article are included in the article's Creative Commons license, unless indicated otherwise in a credit line to the material. If material is not included in the article's Creative Commons license and your intended use is not permitted by statutory regulation or exceeds the permitted use, you will need to obtain permission directly from the copyright holder. To view a copy of this license, visit http://creativecommons.org/licenses/by/4.0/.
\end{abstract}

(C) The Author(s) 2018

\footnotetext{
${ }^{1}$ Condensed Matter Theory Laboratory, RIKEN, Wako, 351-0198 Saitama, Japan. ${ }^{2}$ School of Physics, Georgia Institute of Technology, 30332 Atlanta, GA, USA. ${ }^{3}$ Neutron Scattering Division, Oak Ridge National Laboratory, 37831 Oak Ridge, TN, USA. ${ }^{4}$ NIST Centre for Neutron Research, National Institute of Standards and Technology, 20899 Gaithersburg, MD, USA. ${ }^{5}$ Helmholtz-Zentrum Berlin für Materialien und Energie, D-14109 Berlin, Germany. 6 National High Magnetic Field Laboratory, Florida State University, 32310 Tallahassee, FL, USA. ${ }^{7}$ Department of Physics and Astronomy, University of Tennessee, 37996 Knoxville, TN, USA. ${ }^{8}$ Neutron Scattering Division and Shull-Wollan Center, Oak Ridge National Laboratory, 37831 Oak Ridge, TN, USA. ${ }^{9}$ Key Laboratory of Artificial Structures and Quantum Control, Department of Physics and Astronomy, Shanghai Jiao Tong University, 200240 Shanghai, China. ${ }^{10}$ Collaborative Innovation Center of Advanced Microstructures, 210093 Nanjing, Jiangsu, China. Correspondence and requests for materials should be addressed to Y.K. (email: yoshitomo.kamiya@riken.jp) or to J.M. (email: jma3@sjtu.edu.cn)
} 